\title{
Prognostic significance of the mitochondrial scoring system in ovarian cancer
}

\author{
RYUMA MATSUMOTO $^{1}$, SEIJI ISONISHI ${ }^{1}$, KAZUHIKO OCHIAI ${ }^{1}$, TOMOMI HAMADA ${ }^{2}$, \\ TAKAKO KIYOKAWA ${ }^{2}$, TOSHIAKI TACHIBANA ${ }^{3}$ and HIROSHI ISHIKAWA ${ }^{3}$ \\ Departments of ${ }^{1}$ Obstetrics/Gynecology, ${ }^{2}$ Pathology, and ${ }^{3}$ Anatomy, \\ Jikei University School of Medicine, Tokyo 105-8461, Japan
}

Received May 7, 2010; Accepted June 21, 2010

DOI: $10.3892 /$ etm.2010.118

\begin{abstract}
We report a mitochondrial (MT) scoring system related to response to platinum treatment in ovarian cancer (OC). Ultra-thin sections of surgical specimens of primary tumors prepared from 41 OC patients were examined by electron microscopy. The ovarian carcinoma cell line 2008 and its platinum-resistant variant $\mathrm{C} 13$ were used as controls. Seven independent MT features, including MT diameter, pattern of cresta structure, electron density, MT distribution, pattern of distribution, ovoid ratio and MT architecture, were examined. Each of the seven parameters was assigned a point score of 0-2 and was summed up with a total score of 14. Clinical response and in vitro sensitivity to platinum, taxane, irinotecan and doxorubicin were evaluated. Clinical information was available for 37 of the 41 cases. Twenty-four cases were stage III and, histologically, 16 serous, 6 endometrioid and 6 clear cell carcinoma were included. All of the patients underwent surgery followed by 6 cycles of taxane and platinum chemotherapy. Fifteen patients exhibited a response, while 22 were resistant to treatment. The total MT score was $5.13 \pm 1.13$ $($ mean $\pm \mathrm{SE})$ in the 15 responsive cases and $11.41 \pm 0.43$ in the 22 resistant cases $(\mathrm{P}<0.001)$. Receptor operative characteristic (ROC) analysis revealed that the resistant total 'cut-off' score was $\geq 10$ points $(\mathrm{P}<0.05$; $\mathrm{AUC}=0.86)$ with $95.5 \%$ sensitivity and $80.0 \%$ specificity. The MT scoring system correlated well with response to drugs, with the exception of doxorubicin. The progression-free survival (PFS) curves showed an absolute difference in the 6-month PFS of $10 \%$ (83 vs. 73\%) and in the 12-month PFS of $21 \%$ ( 80 vs. $59 \%$ ), in favor of patients with low MT scores. This MT scoring system correlates very closely with clinical response as well as cellular sensitivity to chemotherapy, resulting in an association with PFS.
\end{abstract}

Correspondence to: Dr Seiji Isonishi, Department of Obstetrics/ Gynecology, Jikei University Daisan Hospital, 4-11-1 Izumi-honnchou, Komae-shi, Tokyo 201-8601, Japan

E-mail: isonishi@jikei.ac.jp

Key words: ovarian cancer, mitochondria, progression-free survival, drug sensitivity

\section{Introduction}

Platinum and taxane are used as two key drugs for the gold standard regimen in ovarian cancer (OC) treatment, although recent innovative treatment strategies have achieved more successful results using an intraperitoneal approach (1) or the dose-dense principle (2). However, platinum resistance remains a major obstacle in cancer therapy. Clinical trials are aimed at circumventing patient relapse within 6 or 12 months after first-line chemotherapy, and new trials are underway to deal with drug resistant OC, including clear-cell and mucinous carcinomas. Recently, we reported that mitochondrial (MT) ultrastructural morphology closely correlates with platinum response in OC (3). We also reported that mitochondria play an important role in platinum sensitivity, as MT-DNA is involved in platinum cell cytotoxicity (4). Furthermore, it has been shown that the stimulation of MT cytochrome $\mathrm{C}$ release results in the enhancement of platinum sensitivity in ovarian carcinoma cells (5). Finally, several studies have shown a direct drug action on mitochondria, inducing the loss of membrane potential and the release of apoptogenic proteins from isolated mitochondria $(6,7)$.

Taxanes are important drugs commonly used to treat OC. Taxane resistance is hard to evaluate in the clinical setting, unlike platinum resistance as determined by GOG criteria, which defines clinical resistance as patient relapse within 6 months of previous platinum chemotherapy. However, basic or translational research studies have addressed the MT-associated mechanisms of paclitaxel resistance, including the association of the Bcl-2 molecule to drug-induced apoptosis $(8,9)$, paclitaxel-induced MT functional abnormality (10), microtubule stabilization associated with MT aggregation (11) and caspase activation of mitrochondria upstream (12). Other investigators have shown MT-dependent apoptosis induced by paclitaxel, etoposide and UV-irradiation (13). Cell cytotoxicity of irinotecan and 5-fluorouracil has also been reported to be related to MT membrane potential (14). These findings indicate that most chemotherapeutic drugs used for OC treatment affect the MT-associated apoptotic cascade; therefore, mitochondria may play a central role in sensitivity to these drugs.

Among all OCs, serous adenocarcinoma is considered to be the most sensitive to standard chemotherapeutic regimens, 
Table I. Scoring system of the mitochondrial ultrastructure.

\begin{tabular}{lccc}
\hline Feature & 0 & 1 & 2 \\
\hline $\begin{array}{l}\text { MT size } \\
\text { (longest diameter) }\end{array}$ & Small & Large \\
Cresta structure & $(\leq 0.7 \mu \mathrm{m})$ & Intermediate & $(\geq 0.8 \mu \mathrm{m})$ \\
Electron density & Clear & Intermediate & Destroyed \\
MT distribution & Low & Intermediate & High \\
$\left(/ 100 \mu \mathrm{m}^{2}\right)$ & High & Intermediate & Low \\
$\begin{array}{l}\text { Distribution pattern } \\
\text { Oval ratio } \\
(\text { short/long) }\end{array}$ & Perinuclear & Intermediate & Dispersed \\
MT type & Short ovoid & Intermediate & Long ovoid \\
$(\geq 0.7)$ & & $(\leq 0.3)$ & Adrenal hepatocyte \\
\hline
\end{tabular}

while other types are less sensitive. Silverberg reported the prognostic significance of a histopathologic grading system of epithelial OC, emphasizing that histopathologic typing is less valuable than grading in predicting survival, but better at predicting tumor responsiveness to chemotherapy agents (15). Malpica et al reported that a two-tier system for grading ovarian serous carcinoma based on nuclear atypia (uniformity vs. pleomorphism) correlated well with the prognosis of survival $(16,17)$. The pathological features of ovarian cancer following chemotherapy have been detailed previously (18).

We recently developed a scoring system of MT morphological findings to determine its potential correlation with responsiveness to chemotherapy, and the application of this system to a series of cases treated in a uniform manner in our institution was analyzed (3). In this study, this scoring system was extended to investigate whether it can be applied to sensitivity to various drugs, histologic type and patient prognosis.

\section{Materials and methods}

Patients. The study group comprised 41 women with advanced stage or recurrent OC who had been treated with primary surgery followed by taxane plus platinum-based chemotherapy at the Department of Obstetrics and Gynecology of Jikei University School of Medicine, Japan, between January 2001 and December 2006. Patients were enrolled in the study after providing informed consent. The study protocol and all accompanying forms and surveys were reviewed and approved by the institutional review board.

Histologic diagnosis. Tumor stage and histologic subtype were determined according to FIGO and WHO guidelines, respectively. All available histological sections were reviewed by two expert pathologists involved in the study. The Gynecologic Oncology Group (GOG) grading system (19) was used in this study.

Clinical evaluation. Clinical examination, serum CA125 assay, chest X-ray, abdominal-pelvic ultrasound and computed tomography scan (CT) were routinely used to evaluate clinical response. Additional investigations were performed when appropriate. Response was characterized according to the
Response Evaluation Criteria in Solid Tumors (RECIST) (20). When no measurable tumor was observed, CA125 response criteria (Gynecologic Cancer Intergroup-Modified Rusting definition) (21) were used. Serum CA125 values were measured immediately before and after chemotherapy.

In vitro drug sensitivity assay. When the appropriate number of viable cells was available from the tumor mass, continuous oxygen consumption (slope of dissolved oxygen; SDO) was monitored with a dissolved oxygen meter. When cells are sensitive to anticancer agents, the number of viable cells decreases in association with the decreased amount of total cellular oxygen consumption. The amount of oxygen consumption correlates with cellular drug sensitivity, and it is a rapid method for assessing chemosensitivity $(22,23)$. The ovarian carcinoma cell line 2008 and its platinum-resistant variant C13 were used as controls. The human cell line 2008 was established from a patient with a serous cystadenocarcinoma of the ovary (24), and a resistant subline C13 was obtained by 13 monthly selections with cisplatin followed by chronic exposure to cisplatin (25).

Electron microscopy. The method of electron microscopy is described in detail in our previous report (3). Briefly, for electron microscopic analysis, cells or samples were fixed in situ with $2.0 \%$ glutalaldehyde. After washing, cells were fixed for $1 \mathrm{~h}$ at $4^{\circ} \mathrm{C}$ in $1 \% \mathrm{OsO}_{4}$. Samples were dehydrated in graded concentrations of ethanol and embedded in Epon 812 epoxy resin. After polymerization, ultra-thin sections were cut parallel to the block surface using a Reichert OUM4 ultramicrotome, stained with uranyl acetate and lead citrate and then examined using a JEOL-1200EX electron microscope at $60 \mathrm{kV}$ acceleration voltage at a magnification of $\mathrm{x} 1,000$, $\mathrm{x} 1,200$ and $\mathrm{x} 2,500$.

By careful examination and comparison of mitochondria in typical platinum-sensitive and -resistant OC cells, we evaluated $\sim 50$ mitochondria and focused on seven independent parameters depicting the most prominent differences between these two representative types of cells: i) MT size (longest diameter: $<0.7 \mu \mathrm{m}, 0.7-0.8,>0.8 \mu \mathrm{m}$ ); ii) Cresta structure (clear, intermediate, destroyed); iii) electron density (low, intermediate, high); iv) MT distribution (no./100 $\mu \mathrm{m}^{2}:<40$, 
Table II. Clinical features of the patients.

\begin{tabular}{|c|c|c|c|c|c|c|}
\hline \multirow[t]{2}{*}{ Characteristic } & \multicolumn{2}{|c|}{ Total } & \multicolumn{2}{|c|}{ Low score } & \multicolumn{2}{|c|}{ High score } \\
\hline & No. & $\%$ & No. & $\%$ & No. & $\%$ \\
\hline No. & 41 & & 16 & & 25 & \\
\hline Age (median) & 51.5 & & 54 & & 47 & \\
\hline \multicolumn{7}{|l|}{ Stage } \\
\hline $\mathrm{I}^{\mathrm{a}}$ & 4 & 9.8 & 3 & 18.8 & 1 & 4.0 \\
\hline II & 2 & 4.9 & 0 & 0.0 & 2 & 8.0 \\
\hline III & 24 & 58.5 & 10 & 62.5 & 14 & 56.0 \\
\hline IV & 5 & 12.2 & 1 & 6.3 & 4 & 16.0 \\
\hline Recurrence & 6 & 14.6 & 2 & 100.0 & 4 & 16.0 \\
\hline \multicolumn{7}{|l|}{ Histology } \\
\hline Serous & 16 & 43.1 & 10 & 62.5 & 6 & 24.0 \\
\hline Mucinous & 1 & 2.3 & 1 & 6.3 & 0 & 0.0 \\
\hline Endometrioid & 6 & 13.6 & 1 & 6.3 & 5 & 20.0 \\
\hline Clear cell & 6 & 13.6 & 1 & 6.3 & 5 & 20.0 \\
\hline Undifferentiated & 2 & 4.5 & 0 & 0.0 & 2 & 8.0 \\
\hline Unclassified & 1 & 11.4 & 1 & 6.3 & 0 & 0.0 \\
\hline Others $^{\mathrm{b}}$ & 9 & 11.4 & 2 & 12.5 & 7 & 28.0 \\
\hline \multicolumn{7}{|l|}{ Tumor grade ${ }^{c}$} \\
\hline 1 & 5 & 31.3 & 1 & 10.0 & 4 & 66.7 \\
\hline 2 & 6 & 37.5 & 4 & 40.0 & 2 & 33.3 \\
\hline 3 & 5 & 31.3 & 5 & 50.0 & 0 & 0.0 \\
\hline \multicolumn{7}{|l|}{ Max. tumor size ${ }^{d}$} \\
\hline$\leq 1 \mathrm{~cm}$ & 28 & 68.3 & 14 & 82.4 & 14 & 58.3 \\
\hline$>1 \mathrm{~cm}$ & 13 & 31.7 & 3 & 17.6 & 10 & 41.7 \\
\hline \multicolumn{7}{|c|}{ No. of residual lesions } \\
\hline$\leq 5$ & 29 & 70.7 & 14 & 82.4 & 15 & 62.5 \\
\hline$>5$ & 12 & 29.3 & 3 & 17.6 & 9 & 37.5 \\
\hline
\end{tabular}

${ }^{\mathrm{a}}$ In vitro assay alone; bincluding sarcoma and other rare tumors; ${ }^{c}$ grading for serous, mucinous and endometrioid; ${ }^{\mathrm{d}}$ maximum size of the residual tumor.

40-80, >80); v) pattern of MT distribution (perinuclear, intermediate, dispersed); vi) ovoid ratio (shortest diameter/longest diameter: $\geq 0.7$, intermediate, $\leq 0.3$ ); vii) $\mathrm{MT}$ architecture (three types: tubular, adrenal or hepatocyte) (Table I). Each of these evaluated parameters was assigned a point score of 0-2, with the exception of MT architecture, which was scored as 0 or 2 . The different features were then summed up for a possible total score of 14 . The 2008 and $\mathrm{C} 13$ cell lines served as examples of typical electron microscopy of drug-sensitive and -resistant cell mitochondria, respectively. In our previous report, the electron density and the MT distribution pattern were selected for the final scoring system based on an epithelial OC (3). However, in this study, non-epithelial ovarian tumors comprised $\sim 25 \%$ of the study population, and the MT scoring was performed using the full set of seven parameters.

Statistical analysis. Differences between samples or groups of samples were determined by the Student's t-test using two-sided P-values. Multiple logistic regression was carried out to investigate the relationship between drug response and the above seven features. Clinical response and in vitro sensi- tivity analysis were compared to the results of the Fisher's exact test. Receiver operator characteristic (ROC) analysis was performed to establish whether an optimal 'cut-off' point could be determined for the MT scoring system and subsequent drug sensitivity.

Progression-free survival (PFS) was calculated from the date of surgery to the date of recurrence as determined by CT or MRI scan. One case with an independent increase of CA125 without detectable tumor was excluded. Kaplan-Meier estimates were used to construct the survival curves (26).

\section{Results}

Patient and tumor characteristics. Patient demographics are summarized in Table II. The median age of the patients was 51 years (range 24-71). According to the FIGO classification, 4 patients had stage I tumors, 2 had stage II, 24 had stage III and 5 had stage IV tumors. In 6 patients, only a recurrent tumor was available for review.

Patients with stage I did not receive chemotherapy, and the remaining 37 cases were analyzed for response to chemo- 
Table III. Results of the scoring system.

\begin{tabular}{lccrrr}
\hline Drug(s) & $\begin{array}{c}\text { Sensitive } \\
\text { mean } \pm \text { SE (no.) }\end{array}$ & $\begin{array}{c}\text { Resistant } \\
\text { mean } \pm \text { SE (no.) }\end{array}$ & P-value & 95\% CI & Cut-off \\
\hline Clinical TC & $5.13 \pm 1.13(15)$ & $11.41 \pm 0.42(22)$ & $<0.0001$ & $3.73-8.82$ & 10 \\
Platinum & $5.75 \pm 1.13(16)$ & $11.60 \pm 0.68(5)$ & 0.0002 & $3.07-8.63$ & 11 \\
Taxane & $4.97 \pm 1.21(12)$ & $10.40 \pm 1.27(10)$ & 0.0030 & $1.75-9.05$ & 5 \\
Irrinotecan & $4.27 \pm 1.13(11)$ & $10.15 \pm 1.53(6)$ & 0.0062 & $1.52-9.94$ & 10 \\
Doxorubicin & $6.31 \pm 1.35(13)$ & $9.00 \pm 1.75(7)$ & 0.1221 & $-2.08-7.46$ & - \\
\hline
\end{tabular}

aResponse to taxane/platinum combination chemotherapy. CI, confidence interval.

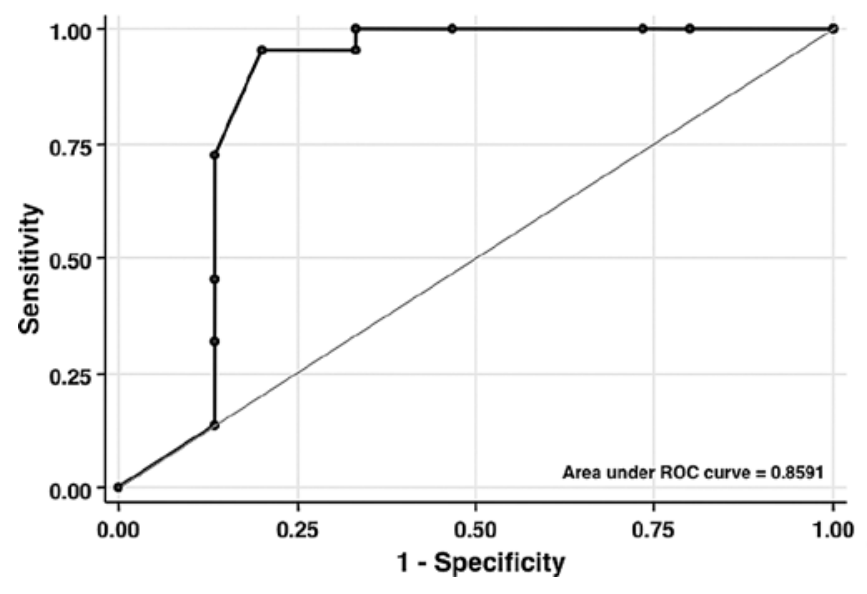

Figure 1. Receiver operator characteristic (ROC) analysis was performed to determine the optimal 'cut-off' score of $\geq 10$ as resistant with sensitivity of $95.5 \%$ and specificity of $89.2 \%$.

therapy. For the recurrent cases, platinum-resistant disease was defined as progression either during therapy or within 6 months of completing therapy with platinum or platinumcontaining chemotherapy. In this study, all recurrent cases had a PFS time of $>6$ months, indicating that they were all platinum-sensitive. Histologically, 19 carcinomas were serous and 6 were endometrioid and clear-cell, respectively. Two were undifferentiated, 1 was mucinous and 1 was unclassified adenocarcinoma. The remaining 9 were other ovarian tumors, including sarcoma, small-cell carcinoma and other rare tumors. Since grading is important for serous and endometrioid carcinoma, these tumors were graded, resulting in a grade of G1 in 5 patients, G2 in 6 and G3 in 5 cases. After surgery, 28 patients had residual disease $\leq 1 \mathrm{~cm}$ and 13 patients had larger residual tumors. Chemotherapy response was not related to the stage, histology, grade, tumor size or residual lesion.

Scoring system for mitochondrial ultrastructure. Seven features were analyzed and scored independently in our grading system. Of the 37 patients receiving post-operative chemotherapy, 15 achieved a response, while 22 were resistant to treatment. The total MT score was $5.13 \pm 1.13$ (mean $\pm \mathrm{SE}$ ) in the 15 responsive cases and $11.41 \pm 0.43$ in the 22 resistant cases $(\mathrm{P}<0.001)$. As shown in Fig. 1, ROC analysis revealed that the resistant total 'cut-off' score was $\geq 10$ points $(\mathrm{P}<0.05$;
AUC $=0.86$ ) with $95.5 \%$ sensitivity, $80.0 \%$ specificity and $89.2 \%$ positive predictive value. Table III lists MT scores for different drugs related to sensitivity. This was highly associated with cellular drug sensitivity, with the exception of doxorubicin. The total scores were in the range of 4-6 in the groups sensitive to platinum, taxane and irinotecan, while they were 10-12 in the resistant groups. ROC analysis revealed that the resistant 'cut-off' scores for platinum, taxane and irinotecan were $\geq 11,5$ and 10 points, respectively.

Based on the above ROC analysis, patients were classified into two groups: patients with low scores $(<10$ points; $n=16)$ or patients with high scores ( $\geq 10$ points; $n=25$ ) (Table II). Most patients in the low-score group corresponded to chemotherapy responders, while patients in the high-score group were from the non-responder group. The distribution, stage and histology were mostly well-balanced between these two groups. The most prognostically relevant clinical features are shown in Table II, including maximum tumor size and the presence of residual disease prior to chemotherapy. Fig. 3 shows the Kaplan-Meier PFS estimates by MT Score. During the median follow-up of 23 months, 11 patients relapsed. The PFS curves show a difference in favor of the low-score group of patients compared to the high-score group (risk ratio 3.99, $\mathrm{P}=0.045$ ), corresponding to an absolute difference in the 6-month PFS of $10 \%$ (83 vs. $73 \%$ ) and in the 12-month PFS of $21 \%$ (80 vs. $59 \%$ ).

\section{Discussion}

Two recent randomized Phase III trials demonstrated that intraperitoneal (1) or weekly dose-dense (2) chemotherapy with carboplatin and paclitaxel resulted in a more favorable survival than standard chemotherapy for OC patients. However, it is not clear whether these two strategies circumvent drug resistance. For the rare type of platinum-resistant OC, such as clear-cell carcinoma or mucinous adenocarcinoma, worldwide clinical trials using combinations other than the platinum/ taxane combination are currently underway. Drug sensitivity (27) or resistance (28) screening prior to chemotherapy may be useful tools when the system relates to the clinical response or prognosis of the patients. An extreme drug-resistant (EDR) test could avoid drug toxicity and the subsequent cost of treatment of patients with highly drug-resistant tumors. EDR tests involve the limited outcome of the elimination of chemotherapy in a resistant tumor $(28,29)$. These tests may correlate 

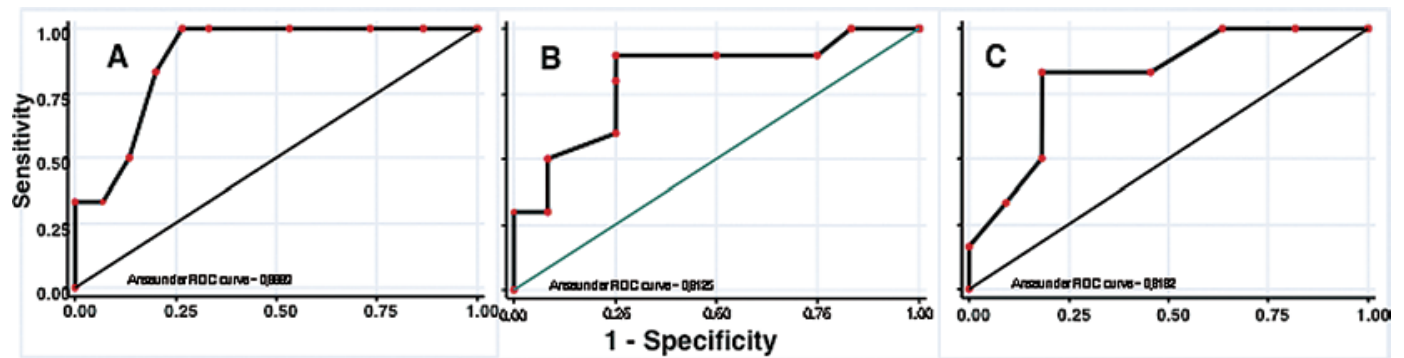

Figure 2. Receptor operative analysis of platinum (A), taxane (B) and irinotecan (C) sensitivity. Resistant 'cut-off' scores for platinum, taxane and irinotecan were $\geq 11,5$ and 10 points, respectively.

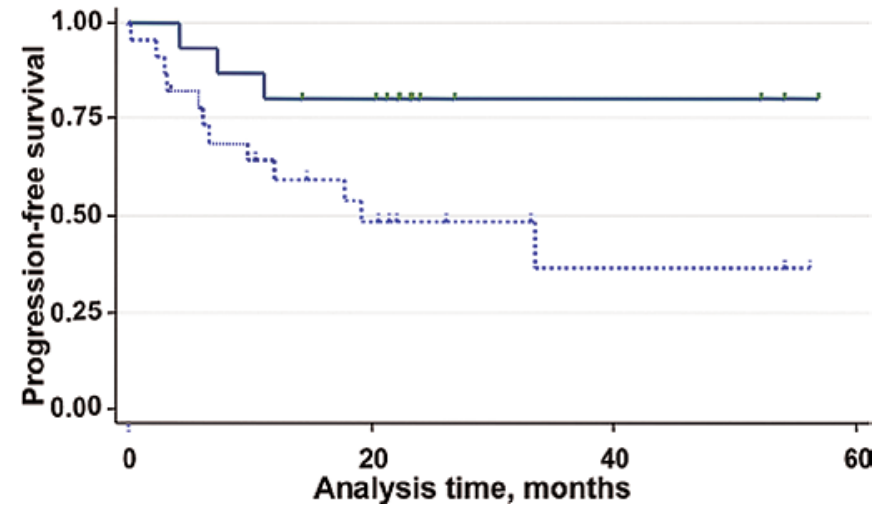

Figure 3. Kaplan-Meier progression-free survival (PFS) estimates by MT score. The PFS curves show a difference in favor of the low-scoring group of patients compared to the high-scoring group (risk ratio $=3.99, \mathrm{P}=0.045$ ).

strongly to our MT scoring system as this assay is not associated with cell survival, but rather measures cellular metabolic activity monitored by MT status. In our previous report, the electron density and MT distribution pattern were selected for the final scoring system based on the platinum sensitivity of epithelial OC (3). However, the present study consisted of 9 non-epithelial ovarian tumors comprising $25 \%$ of the study population, and the scoring system was also applied to nonplatinum agents. MT scoring was performed using the full set of seven parameters.

Here, we report that our MT scoring system is strongly associated with the clinical response to platinum/taxane and is a good predictor of patient response to chemotherapy. However, several issues require discussion.

First of all, the pathologic grading system has been shown to correlate with the survival prognosis in ovarian serous adenocarcinoma (15-17), while histologic type is a good predictor of cellular sensitivity to chemotherapeutic drugs. Our study patients had various types of tumors, including clear-cell carcinoma, mucinous adenocarcinoma, sarcoma and other rare tumors. Mitochondria are cellular ubiquitous organelles and are indispensable organs of the cell involved in controlling respiratory or metabolic functions in tumors of any histologic type or grading. Notably, MT change correlates well with drug sensitivity and with patient survival regardless of the histologic type or grading system. This clearly indicates that the MT scoring system has a clinical advantage over the latter two systems.
Second, the MT scoring system was originally developed on the basis of MT changes in platinum-resistant cells (4). Notably, the system can be applied to taxane- and irinotecanresistant cells, suggesting that MT-impaired cells are resistant to most of the drugs among the standard battery of agents usually selected for the treatment of OC.

This finding is supported by the fact that, in the clinic, platinum-resistant patients are usually resistant to most other chemotherapeutic regimens, including topotecan (30), gemcitabine (31) and weekly taxol (32), as the series of GOG126 trials demonstrated. Our data suggest that a high MT score is a common morphological feature for drug resistance, at least to platinum, taxane and irinotecan. Mitochondria are involved in the mechanism of resistance for the above three drugs. Notably, the resistant 'cut-off' scores for platinum, taxane and irinotecan vary for each drug, suggesting that mitochondria are differentially involved in the mechanism of resistance, although it is not clear how the MT morphological changes relate to its function.

Third, 4 out of 6 recurrent cases showed high MT scores, although more than 6 months had elapsed since the last dose of chemotherapy, suggesting that these cases may have exhibited intrinsic resistance rather than acquired resistance. Unfortunately, the cases did not have any measurable disease at the time of previous chemotherapy, and the response was not evaluated.

Finally, the relevant outcome was based on whether chemotherapy actually resulted in improved survival for the patients in the low-MT score cohort. The data showed significant difference in the 6-month and 1-year PFS. In particular, regarding the 1-year PFS, more than half the patients in the high-score cohort relapsed compared to $20 \%$ in the low-score cohort. This result was consistent with the GOG resistance criteria, defining patient relapse within 6-12 months after the last chemotherapy as a platinum-resistant case. However, there are several other issues to be considered. The MT score highly depends on the condition of the sampled tumor cells. Uncontrollable factors, such as the vascularity of a tumor or the amount of necrosis, limit the extrapolation of the MT scoring system to the clinical results. Data incorporating these factors are not available, while these applications must be considered as speculative at this time. Although the MT scoring system has significant predictive value, any potential role for MT scoring would also vary according to tumor type, the goal of the chemotherapy (adjuvant vs. neoadjuvant or salvage) and the roster of available chemotherapies to choose from. 
The number of cases in our study was small. Carefully designed prospective studies with a larger sample and with clinical follow-up are required to further investigate the clinical relevance of this MT scoring system. However, the data presented here provide evidence that the system is of considerable value as a biomarker for chemosensitivity in OC.

In conclusion, this study showed that the MT scoring system is closely correlated with clinical response as well as cellular sensitivity to platinum, taxane and irinotecan, resulting in the association with PFS.

\section{Acknowledgements}

The authors wish to thank Dr Esther Oliva of Massachusetts General Hospital, Boston, MA, USA, for the critical review of this manuscript.

\section{References}

1. Armstrong D, Bundy B, Wenzel L, Huang H, Baergen R, Lele S, Copeland L, Walker J and Burger R for the Gynecologic Oncology Group: Intraperitoneal cisplatin and paclitaxel in ovarian cancer. N Engl J Med 354: 34-43, 2006.

2. Katsumata N, Yasuda M, Takahashi F, Isonishi S, Jobo T, Aoki D, Tsuda H, Sugiyama T, Kodama S, Kimura E, Ochiai K and Noda $\mathrm{K}$ for the Japanese Gynecologic Oncology Group: Dose-dense paclitaxel once a week in combination with carboplatin every 3 weeks for advanced ovarian cancer; a phase III, open-label, randomized controlled trial. Lancet 374: 1331-1338, 2009.

3. Saitou M, Isonishi S, Hamada T, Kiyokawa T, Tachibana T, Ishikawa $\mathrm{H}$ and Yasuda M: Mitochondrial ultrastructure associated chemotherapy response in ovarian cancer. Oncol Rep 21: 199-204, 2008

4. Hirama M, Isonishi S, Yasuda $M$ and Ishikawa $H$ : Characterization of mitochondria in cisplatin-resistant human ovarian carcinoma cells. Oncol Rep 16: 997-1002, 2006.

5. Isonishi S, Saitou M, Ochiai K, Yasuda $M$ and Tanaka T: Enhancement of sensitivity to cisplatin by orobol is associated with increased mitochondrial cytochrome $\mathrm{c}$ release in human ovarian carcinoma cells. Gynecol Oncol 90: 413-420, 2003.

6. Kim TS, Yun BY and Kim IY: Induction of the mitochondrial permeability transition by selenium compounds mediated by oxidation of the protein thiol groups and generation of the superoxide. Biochem Pharmacol 66: 2301-2311, 2003.

7. Chilin A, Dodoni G, Frezza C, Guiotto A, Barbieri V, Di Lisa F and Canton M: 4-Hydroxymethyl-1,6,8-trimethylfuro [2,3-h] quinolin-2 $(1 \mathrm{H})$-one induces mitochondrial dysfunction and apoptosis upon its intracellular oxidation. J Med Chem 48: 192-199, 2005

8. Ferlini C, Raspaglio G, Mozzetti S, Distefano M, Filippetti F, Martinelli E, Ferrandina G, Gallo D, Ranelletti FO and Scambia G: Bcl-2 down-regulation is a novel mechanism of paclitaxel resistance. Mol Pharmacol 64: 51-58, 2003.

9. Shajahan AN, Wang A, Decker M, Minshall RD, Liu MC and Clarke R: Caveolin-1 tyrosine phosphorylation enhances paclitaxel-mediated cytotoxicity. J Biol Chem 282: 5934-5943, 2007.

10. Flatters SJ and Bennett GJ: Studies of peripheral sensory nerves in paclitaxel-induced painful peripheral neuropathy: evidence for mitochondrial dysfunction. Pain 122: 245-257, 2006.

11. Liu L, Vo A, Liu G and McKeehan WL: Distinct structural domains within C19ORF5 support association with stabilized microtubules and mitochondrial aggregation and genome destruction. Cancer Res 65: 4191-4201, 2005.

12. André N, Carré M, Brasseur G, Pourroy B, Kovacic H, Briand C and Braguer D: Paclitaxel targets mitochondria upstream of caspase activation in intact human neuroblastoma cells. FEBS Lett 532: 256-260, 2002.
13. Matassa AA, Carpenter L, Biden TJ, Humphries MJ and Reyland ME: PKC delta is required for mitochondrial-dependent apoptosis in salivary epithelial cells. J Biol Chem 276: 29719-29728, 2001.

14. Grivicich I, Regner A, da Rocha AB, Grass LB, Alves PA, Kayser GB, Schwartsmann G and Henriques JA: Irinotecan/5fluorouracil combination induces alterations in mitochondrial membrane potential and caspases on colon cancer cell lines. Oncol Res 5: 385-392, 2005.

15. Silverberg SG: Histopathologic grading of ovarian carcinoma: a review and proposal. Intl J Gynecol Pathol 19: 7-15, 2000.

16. Malpica A, Deavers MT, Tornos C, Kurman RJ, Soslow R, Seidman JD, Munsell MF, Gaertner E, Frishberg D and Silva EG: Interobserver and intraobserver variability of a two-tier system for grading ovarian serous carcinoma. Am J Surg Pathol 31: 1168-1174, 2007.

17. Malpica A, Deavers MT, Lu K, Bodurka DC, Atkinson EN, Gershenson DM and Silva EG: Grading ovarian serous carcinoma using a two-tier system. Am J Surg Pathol 28: 496-504, 2004

18. Mc Cluggage WG, Lyness RW, Atkinson RJ, Dobbs SP, Harley I, Mc Clelland HR and Price JH: Morphological effects of chemotherapy on ovarian carcinoma. J Clin Pathol 55: 27-31, 2002.

19. Benda JA and Zaino R: GOG Pathology Manual. Gynecologic Oncology Group, Buffalo, NY, 1994.

20. Therasse P, Arbuck SG, Eisenhauer EA, Wanders J, Kaplan RS Rubinstein L, Verweij J, van Glabbeke M, van Oosterom AT, Christian MC and Gwyther SG: New guidelines to evaluate the response to treatment in solid tumors. European Organization for Research and Treatment of Cancer, National Cancer Institute of the United States, National Cancer Institute of Canada. J Natl Cancer Inst 92: 205-216, 2000.

21. Rustin GJ: Use of CA-125 to assess response to new agents in ovarian cancer trials. J Clin Oncol 21: S187-S193, 2003.

22. Ishikawa T, Zhu BL and Maeda H: Effects of therapeutic agents on cellular respiration as an indication of metabolic activity. Hum Exp Toxicol 25: 135-140, 2006.

23. Amano Y, Okumura C, Yoshida M, Katayama H, Unten S, Arai J, Tagawa T, Hoshina $\mathrm{S}$ and Ishikawa $\mathrm{H}$ : Measuring respiration of cultured cell with oxygen electrode as a metabolic indicator for drug screening. Human Cell 12: 3-10, 1999.

24. Disaida PJ, Sinkovics JG, Rutledge FN and Smith JP: Cellmediated immunity to human malignant cells. Am J Obstet Gynecol 114: 979-989, 1972.

25. Andrews PA, Murphy MP and Howell SB: Differential potentiation of alkylating agent cytotoxicity in human ovarian carcinoma cells by glutathione depletion. Cancer Res 45: 6250-6253, 1985.

26. Parmar MKB and Machin D: Survival Analysis: A Practical Approach. John Wiley \& Sons Inc., New York, pp160-177, 1995.

27. Andreotti PE, Cree IA, Kurbacher CM, Hartmann DM, Linder D, Harel G, Gleiberman I, Caruso PA, Ricks SH, Untch M, Sartori C and Bruckner HW: Chemosensitivity testing of human tumors using a microplate adenosine triphosphate luminescence assay. Clinical correlation for cisplatin resistance of ovarian carcinoma. Cancer Res 55: 5276-5282, 1995.

28. Brown E and Markman M: Tumor chemosensitivity and chemoresistance assays. Cancer 72: 1020-1025, 1996.

29. Tate Thigpen J: Debate; therapy for a patient with platinumresistant/refractory recurrent ovarian cancer should be selected based on results of an in vitro drug sensitivity/resistance assay. Clin Ovarian Cancer 1: 96-110, 2008.

30. Markman M, Blessing JA, DeGeest K, Morgan M, Look KY, Herzog TJ and Rose PG: Lack of efficacy of 24-hour infusional topotecan in platinum-refractory ovarian cancer; a Gynecologic Oncology Group trial. Gynecol Oncol 75: 444-446, 1999.

31. Brewer C, Blessing J, Nagourney R, Morgan M and Hanjani P: Cisplatin plus gemcitabine in platinum-refractory ovarian or primary peritoneal cancer; a phase II study of the Gynecologic Oncology Group. Gynecol Oncol 103: 446-450, 2006.

32. Markman M, Blessing J, Rubin SC, Connor J, Hanjani P and Waggoner S: Phase II trial of weekly paclitaxel $\left(80 \mathrm{mg} / \mathrm{m}^{2}\right)$ in platinum and paclitaxel-resistant ovarian and primary peritoneal cancers; a Gynecologic Oncology Group study. Gynecol Oncol 101: 436-440, 2006 